\title{
Erratum to: 'Sex and History': Talking Sex with Objects from the Past
}

\author{
Kate Fisher, Jen Grove, and Rebecca Langlands
}

\section{Erratum to:}

Chapter 2 in: Louisa Allen et al, (eds), The Palgrave Handbook of Sexuality Education, https://doi.org/10.1057/978-1-137-40033-8_2

The chapter 'Sex and History': Talking Sex with Objects from the Past has now been made available open access under a CC BY 4.0 license. 